\title{
Analysis of genetic population structure of an endangered Serranid fish species in the South Korean waters: a bioinformatic simulation.
}

\author{
Khaled Mohammed-Geba \\ Molecular Biology and Genetic Engineering Division, Department of Zoology, Faculty of Sciences, Menoufia University, Shebin El- Kom, Menoufia, \\ Egypt.
}

\section{ARTICLE INFO}

Article history:

Received on: 17/01/2015

Revised on: 03/02/2015

Accepted on: 15/02/2015

Available online: 27/02/2015

Key words:

Epinephelus akaara, CO1, East China Sea, GenBank, population genetics.

\begin{abstract}
Groupers (Order: Perciformes, Family: Serranidae) are of the most economically important fishes in the world to both fisheries and aquaculture sectors. Several species are now classified as endangered. Red-spotted, Hong Kong grouper, Epinephelus akaara, is a grouper that provides high economic values for the markets in Hong Kong and Japan. This species falls under the International Union for Conservation of the Nature (IUCN) Red List of endangered species. In order to perform a bioinformatic simulating analysis for the genetic population structure of this species in the South Korean waters, more specifically in Namhae island, 73 nucleotide sequences of cytochrome oxidase subunit 1 (CO1) were retrieved from the GenBank database. Number of haplotypes, polymorphic sites, and the interrelationships between haplotypes were all determined. The results indicated the main haplotype lineages in the area of study. Also, signs of recent population expansion could be detected, alongside with identifying some low frequency haplotypes that may have originated as a result of adaptation to the conditions at this area. This study resulted in partitioning of E. akaara population in the Namhae island into several units of interest for conservation.
\end{abstract}

\section{INTRODUCTION}

Species belonging to the family Serranidae represent the most important group of commercial interest for aquaculture and fisheries in the world. It consists of a complex of species collectively named "groupers", inhabiting tropical and subtropical areas in the world, commonly in relation to coral reefs and rocky bottoms. Groupers are known as protogynous hermaphrodites, sexually maturing first as females, and 3-12 years later resorbing their ovarian tissues and developing testicular tissues instead to become functional males.

Their high economic importance and their unique sexual behavior motivated many studies aiming to investigate their genetic diversity, improve growth, and prevent severe diseases [1-7]. The red-spotted grouper Epinephelus akaara is a considerably important economic fish in the Western Pacific, especially in Hong Kong and Japan. Its easy reproduction in captivity increases the future possibilities for aquaculture, but the high mortality rates of larvae make it necessary to optimize its in-hatchery production. The global population of E. akaraa has declined by approximately $63 \%$ over the last 21 years due to high fishing pressure.

\footnotetext{
* Corresponding Author
}

Dr. Khaled Mohammed-Geba, Molecular Biology and Genetic

Engineering Division, Department of Zoology, Faculty of Sciences,

Menoufia University, Shebin El- Kom, Menoufia, Egypt. Tel.: 002-

0482235690,fax : 002-0482235689,e-mail: Khaledspain@yahoo.com
Also, seed capture from the wild is suffering severe decline, putting then the wild populations of the fish in an endangered state [8]. Its fishing efforts are the maximum in Japan, Taiwan, Republic of Korea and southern China. In Hong Kong, it is the most expensive of all groupers available in the market. The great economic importance of this species and its endangered state produced a vast body of research, covering most aspects of E. akaara biology and ecology. Broodstock management and larval rearing techniques are extensively studied in Japan [1]. Sperm cryopreservation for purposes of in-hatchery propagation and conservation could be experimentally achieved and enhanced by several conditions [9]. Moreover, immunity and its molecular bases was a target of extensive research in this species due to the great vulnerability of its hatchery-reared larvae to the fish Nodavirus of the genus Betanodavirus [10]. DNA barcoding, the term that is applied nowadays to the mitochondrial cytochrome $\mathrm{c}$ oxidase sub-unit I (CO1), provides an efficient method for biodiversity assessment as it meets the need for fast, efficient and reliable species identification at this time of climate change and massive habitat destruction. DNA barcoding also has the power to connect different life stages such as eggs, larvae and adults. As such, it can link hundreds of years of taxonomic, ecological, faunistic and ethological studies $[11,12,13]$. This study aims to study the available data about E. akaara in certain area in the Eastern China Sea in order to determine the degree of variability within the population there, as well as to test the effect of some geological events on the relationships between this population and the ones found in other areas in the China Sea. 


\section{MATERIALS AND METHODS}

73 Epinephelus akaara cytochrome oxidase 1 gene (CO1) nucleotide sequences were retrieved from the GenBank database, all are available in the website http://www.ncbi.nlm.nih.gov/nuccore/?term=epinephelus+akaara+ COI. These sequences were solely belonging to the waters of Namhae island in South Korea, from where they were previously sequenced and submitted to GenBank database [14]. The sequences were first aligned using the program clustalX 2.1 [15]. Later on, they were uploaded to the program MEGA6 [16] and aligned using ClustalW [17] in order to calculate the pairwise distances within the population. The alignment was then uploaded to DNAsp 5.0 Software [18] in order to determine the existing haplotypes. The obtained haplotypes were uploaded to the program Network 4.6.1.2 [19] in order to draw median-joining haplotypes network and further demonstrate their interrelationships. The software ARLEQUIN 3.5.1.1 [20] was then applied in order to estimate the D test statistic of Tjima [21] and the Fs statistic of $\mathrm{Fu}$ [22], whose negative values result from the excess of low-frequency haplotypes that arise from selection or rapid population growth $[21,23]$. Recent population expansions as detected by the increasing diversity of haplotypes in a given population and the homogenous patterns of pairwise differences among them were inferred from calculating the index of raggedness, $r$ [24] and R2 parameter [25], using DNAsp software.

The aligned sequences were up-loaded to MEGA6 software. Best DNA substitution model was determined by the ModelTest procedure. Based on this, a neighbor-joining phylogenetic tree was constructed. 1,000 bootstraps were used to enhance the quality of the test.

\section{RESULTS}

Alignment of the 73 nucleotide sequences for E. akaara $\mathrm{CO} 1$ resulted in total common length between all sequences of 633 base-long. Alignment is shown in Figure 1. In this common CO1 zone, 17 polymorhic sites were found (Fig. 1). Pairwise distances were very low, never exceeding 0.0096. Uploading these sequences into the program DNAsp 5.0 resulted in merging the 73 sequences in 18 different haplotypes.

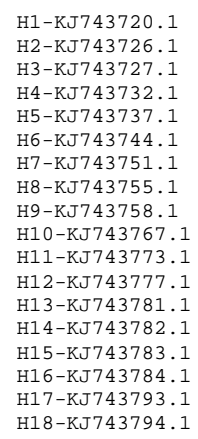

H1-KJ743720.1

H2-KJ743726.1

H3-KJ743727.

$\mathrm{H} 4-\mathrm{KJ} 743732$.

H5-KJ743737.

H6-KJ743744.1

H7-KJ743751.1

H8-KJ743755.

H9-KJ743758.1

H10-KJ743767.1

$\mathrm{H} 11-\mathrm{KJ} 743773$.

H12-KJ743777.

H13-KJ743781.

H14-KJ743782.1

H15-KJ743783.1

H16-KJ743784.1

H17-KJ743793.

H18-KJ743794.1

$\mathrm{H} 1-\mathrm{KJ} 743720.1$ H2-KJ743726.1 H3-KJ743727. 1 $\mathrm{H} 4-\mathrm{KJ} 743732.1$ H5-KJ743737.1 6-KJ743744.1 H7-KJ743751. H8-KJ743755. H9-KJ743758.1 H10-KJ743767. H11-KJ743773. H12-KJ743777.1 H13-KJ743781.1 H14-KJ743782.1 H15-KJ743783.1 H16-KJ743784.1 H17-KJ743793.1 H18-KJ743794.1
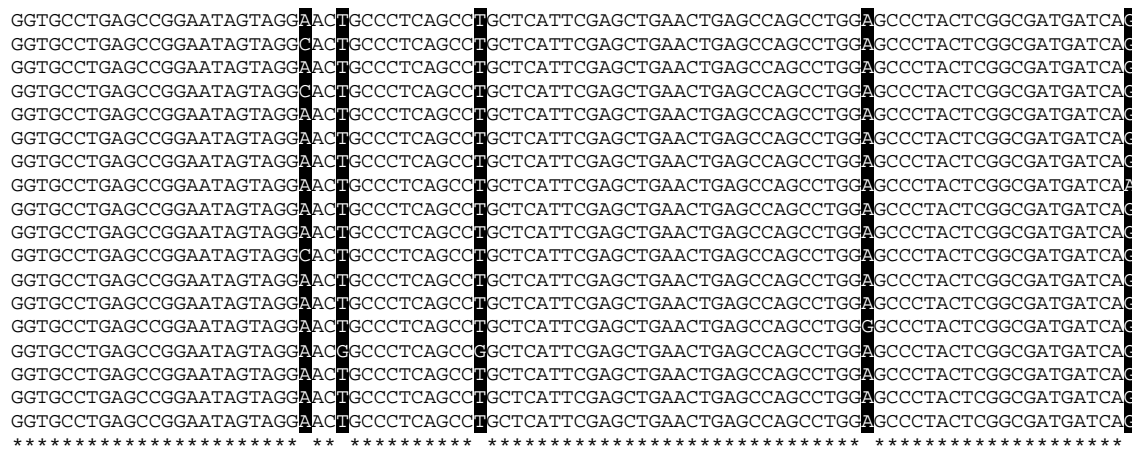

TCTATAATGTAATTGTTACAGCACATGCT

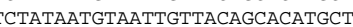
TCTATAATGTAATTGTTACAGCACATGCT TCTATAATGTAATTGTTACAGCACATGCT TCTATAATGTAATTGTTACAGCACATGCT TCTATAATGTAATTGTTACAGCACATGCT TCTATAATGTAATTGTTACAGCACATGCT TCTATAATGTAATTGTTACAGCACATGCT TCTATAATGTAATTGTTACAGCACATGCT TCTATAATGTAATTGTTACAGCACATGCT TCTATGATTACACCACA TIATAIAATITACACATGCI TCACATGCI CTATAATGTAATTGTTACAGCACATGCT 促 TCTATAATGTAATTGTTACAGCACATGCT TCTATAATGTAATTGTTACAGCACATGCT ATCTATAATGTAATTGTTACAGCACATGCT ATCTATAATGTAATTGTTACAGCACATGCT

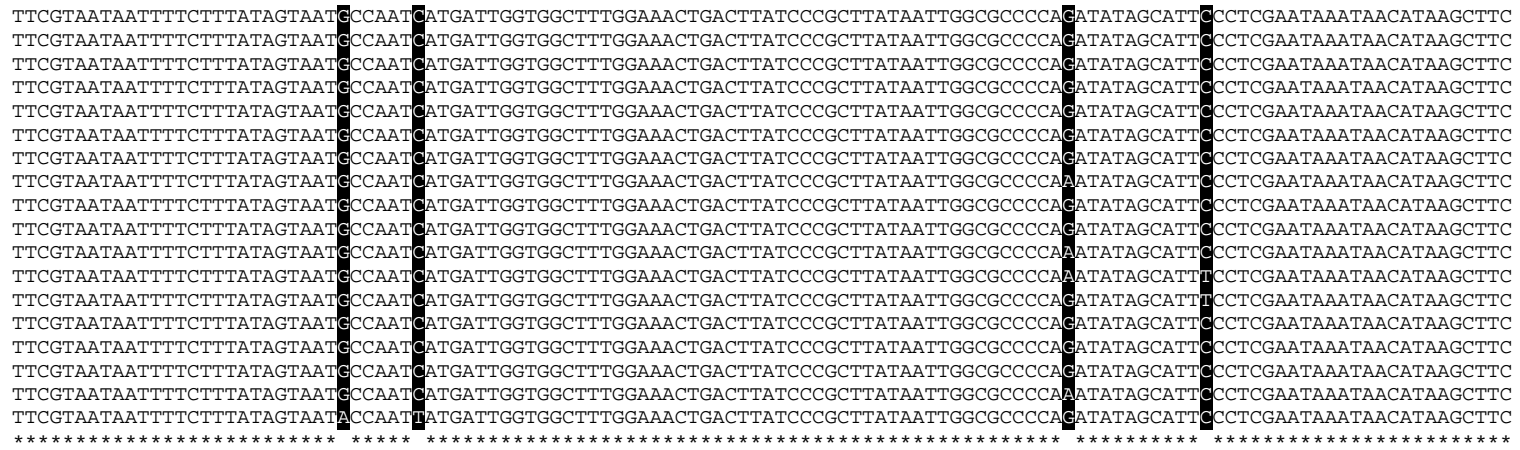
TGACTCCTCCCCCCATCCTTCCTGCTTCTTCTAGCCTCTTCTGGGGTAGAAGCCGGCGCTGGTACTGGTTGAACAGTTTACCCCCCTCTAGCAGGAAACCTGGCCCATGCAGGTGCATCT 作 TTCCCCCATCCTTCCTGCTTCTTCTAGCCTCTTCTGGGGTAGAAGCCGGCGCTGGTACTGGTTGAACAGTTTACCCCCCTCTAGCAGGAAACCTGGCCCATGCAGGTGCATCT CTCCCCCCATCCTTCCTGCTTCTTCTAGCCTCTTCTGGGGTAGAAGCCGGCGCTGGTACTGGTTGAACAGTTTACCCCCCTCTAGCAGGAAACCTGGCCCATGCAGGTGCATCT CTCCCCCCATCCTTCCTGCTTCTTCTAGCCTCTTCTGGGGTAGAAGCCGGCGCTGGTACTGGTTGAACAGTTTACCCCCCTCTAGCAGGAAACCTGGCCCATGCAGGTGCATCT CTCCCCCCATCCTTCCTGCTTCTTCTAGCCTCTTCTGGGGTAGAAGCCGGCGCTGGTACTGGTTGAACAGTTTACCCCCCTCTAGCAGGAAACCTGGCCCATGCAGGTGCATCT TTCCCCCATCCTTCCTGCTTCTTCTAGCCTCTTCTGGGGTAGAAGCCGGCGCTGGTACTGGTTGAACAGTTTACCCCCCTCTAGCAGGAAACCTGGCCCATGCAGGTGCATCT CTCCCCCCATCCTTCCTGCTTCTTCTAGCCTCTTCTGGGGTAGAAGCCGGCGCTGGTACTGGTTGAACAGTTTACCCCCCTCTAGCAGGAAACCTGGCCCATGCAGGTGCATCT

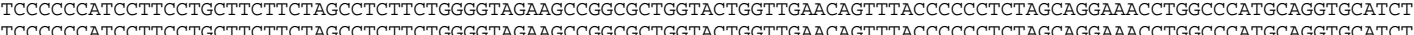
TTCCCCCATCCTTCCTGCTTCTTCTAGCCTCTTCTGGGGTAGAAGCCGGCGCTGGTACTGGTTGAACAGTTTACCCCCCTCTAGCAGGAAACCTGGCCCATGCAGGTGCATCT
CTCCCCCATCCTTCCTGCTTCTTCTAGCCTCTTCTGGGGTAGAAGCCGGCGCTGGTACTGGTTGAACAGTTTACCCCCCTCTAGCAGGAAACCTGGCCATGCAGGTGCATCT CTCCCCCCATCCTTCCTGCTTCTTCTAGCCTCTTCTGGGGTAGAAGCCGGCGCTGGTACTGGTTGAACAGTTTACCCCCCTCTAGCAGGAAACCTGGCCCATGCAGGTGCATCT (⿸丆口

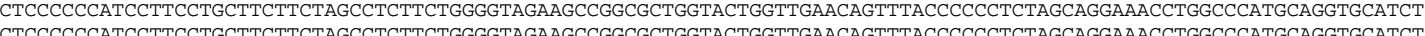
TGACT CCTCCCCCCATCCTTCCTGCTTCTTCTAGCCTCTTCTGGGGTAGAAGCCGGCGCTGGTACTGGTTGAACAGTTTACCCCCCTCTAGCAGGAAACCTGGCCCATGCAGGTGCATCT 


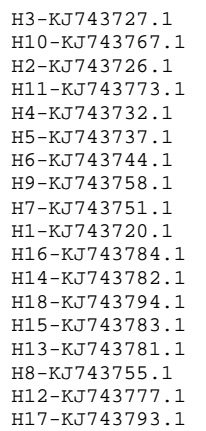

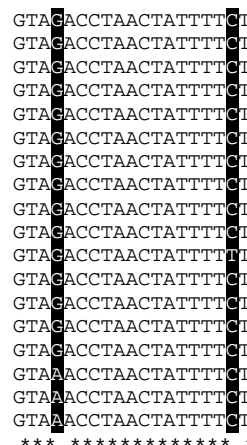

CCTACATCTTGCAGGGATTTCATCAATTCTAGGGGCAATTAACTTCATTACAACCATCATCAACATGAAACCCCCCGCCACCTCTCAGTATCA 促 TCCCTACATCTTGCAGGGATTTCATCAATTCTAGGGGCAATTAACTTCATTACAACCATCATCAACATGAAACCCCCCGCCACCTCTCAGTATCA CCTACATCTTGCAGGGATTTCATCAATTCTAGGGGCAATTAACTTCATTACAACCATCATCAACATGAAACCCCCCGCCACCTCTCAGTATCA 作 CCCTACATCTTGCAGGGATTTCATCAATTCTAGGGGCAATTAACTTCATTACAACCATCATCAACATGAAACCCCCCGCCACCTCTCAGTATCA CCCTACATCTTGCAGGGATTTCATCAATTCTAGGGGCAATTAACTTCATTACAACCATCATCAACATGAAACCCCCCGCCACCTCTCAGTATCA

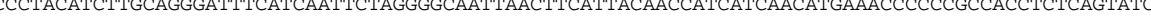
CCCTACATCTTGCAGGGATTTCATCAATTCTAGGGGCAATTAACTTCATTACAACCATCATCAACATGAAACCCCCCGCCACCTCTCAGTATCA CCCTACATCTTGCAGGGATTTCATCAATTCTAGGGGCAATTAACTTCATTACAACCATCATCAACATGAAACCCCCCGCCACCTCTCAGTATCA CCCTACATCTTGCAGGGATTTCATCAATTCTAGGGGCAATTAACTTCATTACAACCATCATCAACATGAAACCCCCCGCCACCTCTCAGTATC CCCTACATCTTGCAGGGATT

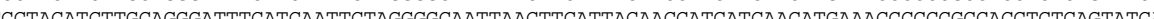

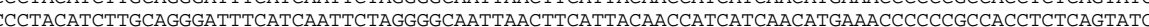
CCCTACATCTTGCAGGGATTTCATCAATTCTAGGGGCAATTAACTTCATTACAACCATCATCAACATGAAACCCCCCGCCACCTCTCAGTATC

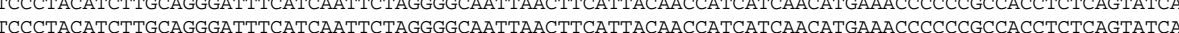
CCCTACATCTTGCAGGGATTTCATCAATTCTAGGGGCAATTAACTTCATTACAACCATCATCAACATGAAACCCCCCGCCACCTCTCAGTATCA
CCCTACATCTTGCAGGGATTTCATCAATTCTAGGGGAATTAACTTCATTACAACCATCATCAACATGAAACCCCCGCCACCTCTCAGTATCA CCCTACATCTTGCAGGGATTTCATCAATTCTAGGGGCAATTAACTTCATTACAACCATCATCAACATGAAACCCCCCGCCACCTCTCAGTATCA $A C A C C A$
$A C A C C A$
$A C A C C C$
$A C A C C C$
$A C A C C C$
ACACCC
ACACCC
ACACCC
ACACCA
ACACCC
ACACCC
ACACCC
ACACCC
ACACCC
ACACCC
ACACCC
ACACCA H2 - KJ 743726.1 H3-KJ743727.1 H4-KJ743732.1 H5-KJ743737.1 H6-KJ743744.1 H7-KJ743751.1 H8-KJ743755.1 H9-KJ743758.1 H10-KJ743767.1 H11-KJ743773. H12-KJ743777. H13-KJ743781.1 H13-KJ743781. H14-KJ743782. H15-KJ743783. H16-KJ743784. H17-KJ743793.

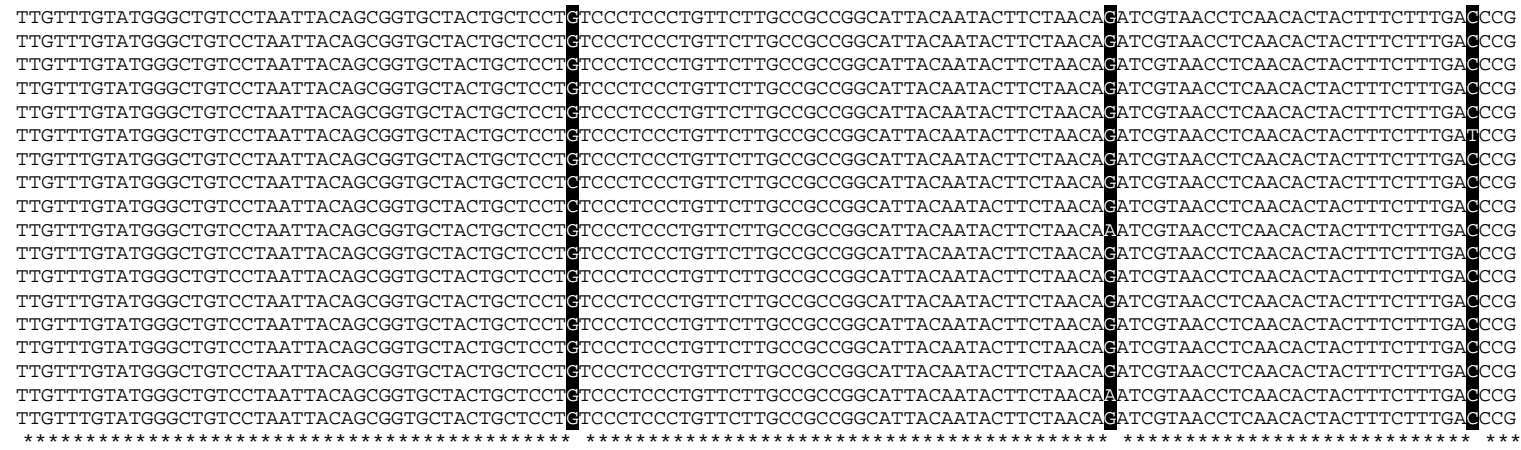

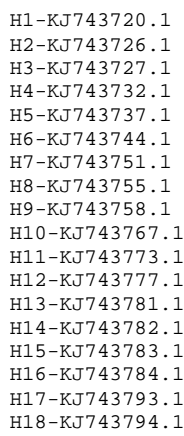

GCCGGAGGGGGAGATCCTATCCTTTACCAACAC GCCGGAGGGGGAGATCCTATCCTTTACCAACAC GCCGGAGGGGGAGATCCTATCCTTTACCAACAC GCCGGAGGGGGAGATCCTATCCTTTACCAACAC GCCGGAGGGGAGATCCTAICCTITACCAACAC GCCGGAGGGGAGATCCTATCCTTTACCAACAC GCGGAGGGGGAGATCCTATCCIITACCAACAC GCCGGAGGGGGAGATCCTATCCTTTACCAACAC GCCGGAGGGGGAGATCCTATCCTTTACCAACAC GCCGGAGGGGGAGATCCTATCCTTTACCAACAC GCCGGAGGGGGAGATCCTATCCTTTACCAACAC GCCGGAGGGGGAGATCCTATCCTTTACCAACAC GCCGGAGGGGGAGATCCTATCCTTTACCAACAC GCCGGAGGGGGAGATCCTATCCTTTACCAACAC GCCGGAGGGGGAGATCCTATCCTTTACCAACAC GCCGGAGGGGAGATCCTATCCTTTACCAACAC CCCGAGGGGAGATCCIATCCITTACCAACAC GCGGAGGGAGATCIAICCTITACCAACAC GCCGGAGGGGGAGATCCTATCCTTTACCAACAC

Fig. 1: Clustal X alignment for E. akaara CO1 sequence haplotypes (H1-H18). White letters over black background mark the polymorphic nucleotide sites.

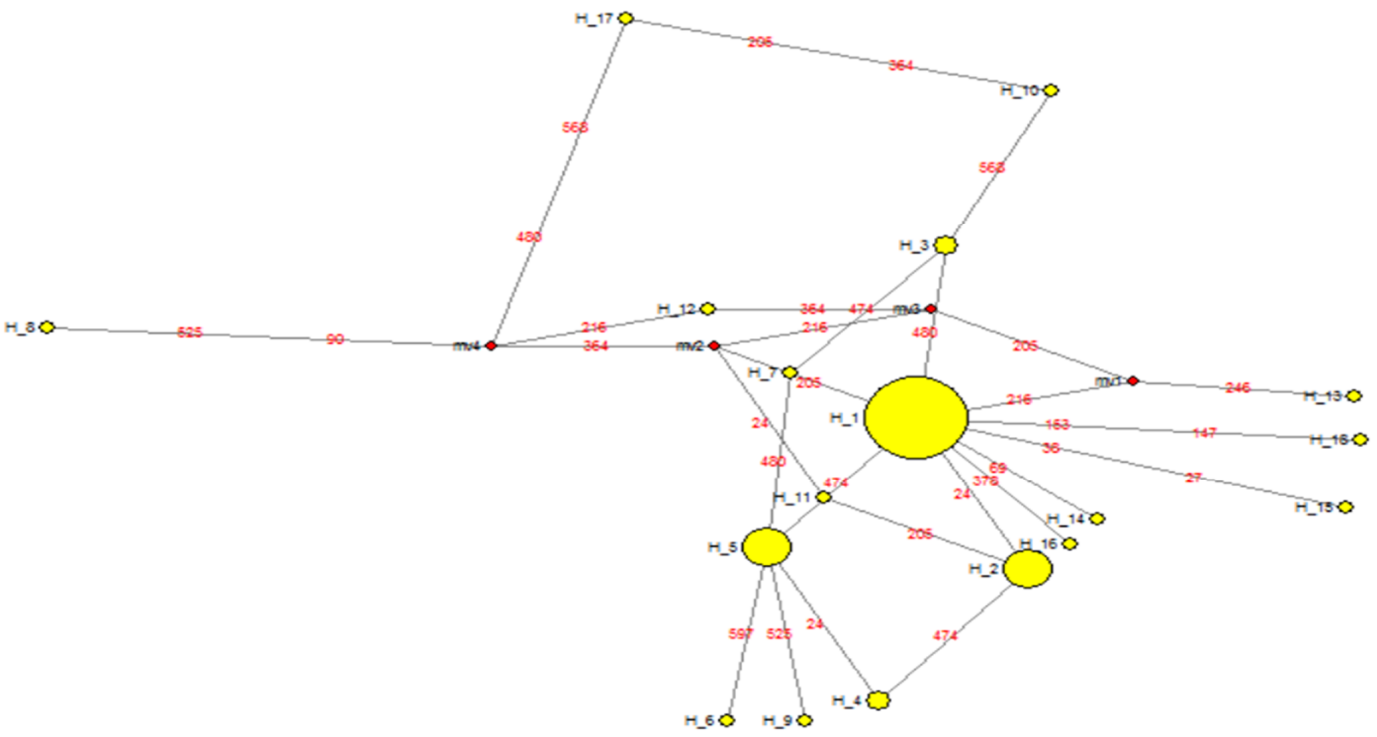

Fig. 2: Median joining network constructed for different haplotypes of E. akaara in Namhae island. Size of each yellow circle is proportional to the number of individuals belonging to a given haplotypes represented by the circle. Haplotypes 1 (H1), 2, 5 are shown as greater circles. Other low-number and singletone haplotypes are shown as the minor circles. 


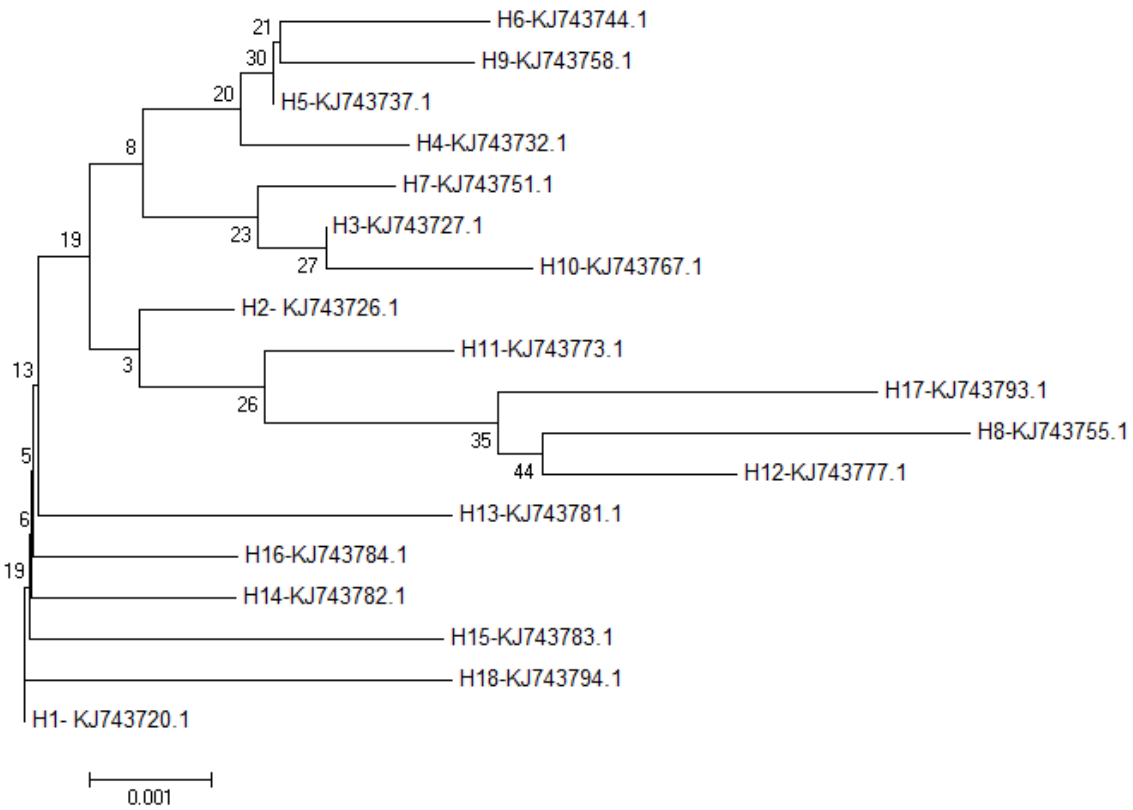

Fig. 3: Neighbor-Joining tree constructed between the 18 haplotypes found for E. akaara CO1 gene, rooted to the ancestral haplotype H1.

Haplotype 1 (H1) included 38 sequences with the following GenBank accession numbers: KJ743720.1, KJ743721.1, KJ743722.1, KJ743728.1, KJ743730.1, KJ743731.1, KJ743733.1, KJ743734.1, KJ743735.1, KJ743736.1, KJ743738.1, KJ743739.1, KJ743741.1, KJ743742.1, KJ743743.1, KJ743745.1, KJ743748.1, KJ743749.1, KJ743750.1, KJ743753.1, KJ743759.1, KJ743760.1, KJ743766.1, KJ743768.1, KJ743771.1, KJ743775.1, KJ743776.1, KJ743778.1, KJ743780.1, KJ743785.1, KJ743786.1, KJ743787.1, KJ743790.1, KJ743789.1, KJ743791.1, KJ743792.1, KJ743795.1, $K J 743796.1$. Haplotype 2 (H2) included the sequences with the 9 following accession numbers: KJ743726.1, KJ743729.1, KJ743747.1, KJ743756.1, KJ743761.1, KJ743765.1, KJ743772.1, KJ743774.1, KJ743788.1. Haplotype 5 (H5) included the 9 sequences with the following accession numbers: KJ743737.1, KJ743740.1, KJ743754.1, KJ743757.1, KJ743762.1, KJ743763.1, KJ743764.1, KJ743769.1, KJ743770.1. Haplotype 3 (H3) included the 2 sequences with the following accession numbers: KJ743727.1, KJ743779.1. Haplotype 4 (H4) included the 2 sequences with the following accession numbers: KJ743732.1, KJ743752.1. Haplotypes from 6 to 18 (H6-H18) were only represented by singletone sequences, belonging to the accession numbers: KJ743744.1, KJ743751.1, KJ743755.1, KJ743758.1, KJ743767.1, KJ743773.1, KJ743777.1, KJ743781.1, KJ743782.1, KJ743783.1, KJ743784.1, KJ743793.1, KJ743794.1.

Haplotypes Median Joining Network drawn by the program Network 4.6.1.2 showed an intricate system with a starlike distribution around the haplotype $1(\mathrm{H} 1)$, which can be then considered as the main lineages for E. akaara in Namhae waters. Two more haplotypes (H2, H5) are also well-established and form bases for radiation for the other haplotypes less commonly distributed in the area (Figure 2).
Demographic parameters used indicated all a recent expansion. Significantly negative D value of Tajima $(-1.83923, \mathrm{p}=0.00900)$ and $\mathrm{Fs}$ of $\mathrm{Fu}(-14.05508, \mathrm{p}=0.0000)$ were found. Furthermore, mismatch analyses suggest a strong possibility of recent population expansion as inferred from the non-significant raggedness (0.81000) and the low R2 value (0.04225). Finally, the genealogical relationships between the 17 obtained haplotypes are shown in the neighbor-joining tree presented in Figure 3.

\section{DISCUSSION}

Despite the threats E. akaara is facing in its natural habitats, this species in Namhae waters seem to be well-adapted and with marks of recent expansion. The presence of several haplotypes provides a special importance for the stock of this species in that area due to the presence of several conservation units. Trials of broodstock development should take into account this haplotypes diversity for keeping the integrity of their natural equilibrium. The relatedness between the main haplotypes (H1) and the other haplotypes with lower frequencies may indicate some local adaptation of in the area.

Structuring of E. akaara populations in China Sea is an attractive topic, despite receiving very little attention from the population genetics viewpoint. Higher nucleotide diversities were found in E. akaara in Northern populations of the China Sea than in Southern populations [26]. The areas covered by the previous study [26] were to the South of Namhae island from which the samples of E. akaara, to which the sequences in the current study belong, were obtained [14]. However, the low nucleotide diversity index $(\pi=0.00208)$ for E. akaara in Namhae island, coupled to the low raggedness values found in this study among COI sequences may refer to that the effect of the low seawater level in the last 
glacial maximum (130 meters below the level of today) not only affected E. akaara in the continental shelf of East and South China sea, but also extended to Namhae region. This possibly leads to a conclusion that this species suffered an old bottle-nick effect followed by a recent extension. No similar studies for E. akaara in this region were found, except the one mentioned before [26], that applied the sequence of the mitochondrial control region for their investigation. However, South and Eastern China Sea represented a key area for fish population genetic studies, using different mitochondrial markers in several species. Several studies pointed to the impacts of climatic and seawater levels oscillations in the Pleistocene in East and South Asian seas. Populations of crimson snapper Lutjanus erythropterus in China Sea had possibly experienced some bottleneck effect followed by population expansion since the late Pleistocene [27]. The mud crab Scylla paramamosain populations thriving along the Chinese coast also seem to have similar bottleneck and recent population expansion [28]. However, no signs of population bottlenecks were found in the pelagic, migratory species found in the same area, the mackerel Scomber japonicus, despite being a target for excessive fishing and habitat destruction [29]. Another school-forming fish, the Fourfinger threadfin, Eleutheronema tetradactylum, did not show any signs for recent population expansion [30]. Therefore, marine species living in the East Asia responded differentially to the lowering of sea levels during the Pleistocene, with the effect is more profound over reef-dwelling fish species. The high market popularity of the groupers in the international markets and the sincere trials for optimization of their aquaculture requires more work for characterization of different lineages and genealogy, especially for detection of species and individuals with good degree of adaptation to the environment where they will be introduced. From the ecological viewpoint, characterization of different units of conservation is a crucial subject, especially when a species facing population declining and a plausible risk of extinction is concerned.

\section{ACKNOWLEDGMENT}

The author would like to appreciate his deep thanks to Doctros. S.H. Han, Y.D. Lee, H.J. Baek, H.S. Oh, and C.H. Noh for making the sequences of E. akaara $\mathrm{CO} 1$ gene available in the GenBank database, what was fundamental for compliance of this study. Also, I would like to appreciate the sincere efforts of the anonymous reviewers and editors of the Journal of Applied Biology \& Biotechnology in enhancing the quality of this work by their suggestions.

\section{REFERENCES}

1. Okumura S, Okamoto K, Oomori R, Nakazono A. Spawning behavior and artificial fertilization in captive reared red spotted grouper, Epinephelus akaara. Aquaculture 2002; 206: 165-173. DOI:10.1016/S0044-8486(01)00722-0.

2. Heemstra PC, Randall JE. Groupers of the World (Family Serranidae, Subfamily Epinephelinae). An annotated and illustrated catalogue of the grouper, rockcod, hind, coral grouper and lyretail species known to date. FAO Species Catalogue 1993; 125: pp. 106-107.

3. Antoro S, Na-Nakorn U, Koedprang W. Study of genetic diversity of orange-spotted grouper, Epinephelus coioides, from Thailand and Indonesia using micro-satellite markers. Mar Biotechnol 2006; 8: 17-26.

4. Goldstein RJ. Marine Reef Aquarium Handbook $2^{\text {nd }}$ Edition. Chapter 22: Fishes: care and breeding. Barron's Educational Series, Inc. 2006, p. 168.

5. Heppell SS, Heppell SA, Coleman FC, Koenig CC. Models to compare management options for a protogynous fish. Ecol Appl 2006; 16: 238-249. DOI: 10.1890/04-1113.

6. Liu W, Hsu CH, Chang CY, Chen HH, Lin CS. Immune response against grouper necrosis virus by vaccination of virus-like particles. Vaccine 2006; 24: 6282-6287. DOI:10.1016/j.vaccine.2006.05.073.

7. Zhou G-Z, Li Z-Q, Yuan X-P, Zhang Q-Y. Establishment, Characterization, and Virus Susceptibility of a New Marine Cell Line from Red Spotted Grouper (Epinephelus akaara). Mar Biotechnol 2007; 9: 370-376. DOI: 10.1111/j.1365-2761.2011.01281.x.

8. Cornish, A 2003. Epinephelus akaara. The IUCN Red List of Threatened Species. Version 2014.2. <www.iucnredlist.org>. Downloaded on 07 September 2014.

9. He Q, Lu G, Che K, Zhao E, Fang Q, Wang H, Liu J, Huang C, Dong Q. Sperm cryopreservation of the endangered red spotted grouper, Epinephelus akaara, with a special emphasis on membrane lipids. Aquaculture 2011; 318: 185-190. DOI:10.1016/j.aquaculture.2011.05.025.

10. Mao M-G, Jiang J-L, Perálvarez-Marín A, Wang K-J, Lei J-L. Characterization of the $\mathrm{Mx}$ and hepcidin genes in Epinephelus akaara asymptomatic carriers of the nervous necrosis virus. Aquaculture2013; 408-409: 175-183. DOI:10.1016/j.aquaculture.2013.05.039.

11. Hebert PDN, Cywinska A, Ball S, deWaard J. Biological identifications through DNA barcodes. Proceed Royal Soc London B 2003a; 270: 313-321. DOI:10.1098/rspb.2002.2218.

12. Hebert PDN, Ratnasingham S, deWaard J. Barcoding animal life: cytochrome c oxidase subunit 1 divergences among closely related species. Proceed Royal Soc London B 2003b; 270: 96-99. DOI: 10.1098/rsbl.2003.0025.

13. Hendrich L, Morinière J, Haszprunar G, Hebert PD, Hausmann A, Köhler, F, Balke M. A comprehensive DNA barcode database for Central European beetles with a focus on Germany: adding more than 3500 identified species to BOLD. Mol Ecol Resour 2014; DOI: 10.1111/1755-0998.12354.

14. Han S-H, Lee Y-D, Baek H-J, Oh H-S, Noh C-H. 2014. Genetic structure of the Hong Kong grouper (Epinephelus akaara) based on the mitochondrial COI haplotypes in South Korea. Direct submission to GenBank database.

15. Larkin MA, Blackshields G, Brown NP, Chenna R, McGettigan PA, McWilliam H, Valentin F, Wallace IM, Wilm A, Lopez R, Thompson JD, Gibson TJ, Higgins DG. ClustalW and ClustalX version 2.0. Bioinformatics 2007; 23: 2947-8.

16. Tamura K, Stecher G, Peterson D, Filipski A, Kumar S. MEGA6: Molecular Evolutionary Genetics Analysis Version 6.0. Molecular Biology and Evolution 2013; 30: 2725-2729. DOI: 10.1093/molbev/mst 197 .

17. Thompson JD, Higgins DG, Gibson TJ. CLUSTAL W: improving the sensitivity of progressive multiple sequence alignment through sequence weighting, position-specific gap penalties and weight matrix choice. Nuc Acid Res 1994; 22: 4673-4680.

18. Rozas, J S-D, Juan C, Messeguer X, Rozas R. DnaSP, DNA polymorphism analyses by the coalescent and other methods. Bioinformatic 2003; 19: 2496-2497. DOI: 10.1093/bioinformatics/btg359.

19. Bandelt H-J, Peter F, Röhl A. Median-joining networks for inferring intraspecific phylogenies. Mol Biol Evol 1999; 16: 37-48.

20. Excoffier L, Lischer HEL. Arlequin suite ver 3.5: a new series of programs to perform population genetics analyses under Linux and Windows. Mol Ecol Res 2010; 10: 564-567. DOI: 10.1111/j.17550998.2010.02847.x. 
21. Tajima F. Statistical method for testing the neutral mutation hypothesis by DNA polymorphism. Genetics 1989; 123: 585-595.

22. Fu YX. Statistical tests of neutrality of mutations against population growth, hitchhiking and background selection. Genetics 1997; 147: 915-25.

23. Borrell YJ, Piñera JA, Sánchez-Prado JA, Blanco G. Mitochondrial DNA and microsatellite genetic differentiation in the European anchovy Engraulis encrasicolus L. ICES J Mar Sci 2012; 69: 13571371.

24. Harpending HC. Signature of ancient population growth in a lowresolution mitochondrial DNA mismatch distribution. Human Biol: 591-600.

25. Ramos-Onsins SE, Rozas J. Statistical properties of new neutrality tests against population growth. Mol Biol Evol. 2002; 19: 2092-100.

26. Chen S, Liu T, Li Z, Gao T. Genetic population structuring and demographic history of red spotted grouper (Epinephelus akaara) in South and East China Sea. African J Biotechnol 2008; 7: 3554-3562.

27. Zhang J, Cai Z, Huang L. Population genetic structure of crimson snapper Lutjanus erythropterus in East Asia, revealed by analysis of the mitochondrial control region. ICES J Mar Sci 2006; 63: 693-704. DOI:10.1016/j.icesjms.2006.01.004.

28. He L, Zhang A, Weese D, Zhu C, Jiang C, Qiao Z. Late Pleistocene population expansion of Scylla paramamosain along the coast of China: A population dynamic response to the Last Interglacial sea level highstand. J Experiment Mar Biol Ecol 2010; 385: 20-28. DOI:10.1016/j.jembe.2010.01.019.
29. Zeng L, Cheng Q, Chen X. Microsatellite analysis reveals the population structure and migration patterns of Scomber japonicus (Scombridae) with continuous distribution in the East and South China Seas. Biochem System Ecol 2012; 42: 83-93. DOI:10.1016/j.bse.2012.02.014.

30. Wang J, Sun P, Yin F. Low mtDNA Cytb diversity and shallow population structure of Eleutheronema tetradactylum in the East China Sea and the South China Sea. Biochem System Ecol 2014; 55: 268-274. DOI:10.1016/j.bse.2014.03.026.

\section{How to cite this article:}

Khaled Mohammed-Geba. Analysis of genetic population structure of an endangered Serranid fish species in the South Korean waters: a bioinformatic simulation. J App Biol Biotech, 2015; 3 (01): 024-029. 